\title{
PRINCIPAL TOROIDAL BUNDLES OVER CAUCHY-RIEMANN PRODUCTS
}

\author{
L. MARIA ABATANGELO \\ SORIN DRAGOMIR \\ Università degli Studi di Bari - Dipartimento di Matematica \\ Trav.200 via Re David n.4, 70125 BARI ITALY
}

(Received December 13, 1988)

\begin{abstract}
The main result we obtain is that given $\pi: \mathrm{N} \rightarrow \mathrm{M}$ a $\mathrm{T}^{\mathrm{s}}$-subbundle of the generalized Hopf fibration $\bar{\pi}: \mathrm{H}^{2 \mathrm{n}+3} \rightarrow \mathbb{C} \mathrm{P}^{\mathrm{n}}$ over a Cauchy-Riemann product $i: M \subseteq \mathbf{C P}^{\mathbf{n}}$, i.e. $\mathbf{j}: \mathbf{N} \subseteq \mathrm{H}^{2 \mathrm{n}+\mathrm{s}}$ is a diffeomorphism on fibres and $\bar{\pi} \circ \mathrm{j}=\mathrm{i} \circ \pi$, if $\mathrm{S}$ is even and $\mathrm{N}$ is a closed submanifold tangent to the structure vectors of the canonical $\mathscr{R}$ structure on $\mathrm{H}^{2 \mathrm{n}+\mathrm{s}}$ then $\mathrm{N}$ is a Cauchy-Riemann submanifold whose Chen class is non-vanishing.
\end{abstract}

KEY WORDS AND PHRASES. Principal toroidal bundle, $\mathscr{R}$-manifold, generalized Hopf fibration, framed C.R. submanifold, characteristic form.

1980 MATHEMATICS SUBJECT CLASSIFICATION CODE: 53C40,53C55

\section{1.- INTRODUCTION AND STATEMENT OF RESULTS.}

As a tentative of unifying the concepts of complex and anti-invariant submanifolds of an almost Hermitian manifold, A. BEJANCU, [1], has introduced the notion of Cauchy-Riemann (C.R.) submanifold. This has soon proved to possess a largely rich number of geometrical properties; e.g. by a result of D.E.BLAIR \& B. Y.CHEN, [2], any C.R. submanifold of a Hermitian manifold is a Cauchy-Riemann manifold, in the sense of A.ANDREOTTI \& C.D.HILL, [3].

Let $M^{2 n+s}$ be a real $(2 n+s)$-dimensional manifold carrying a metrical f-structure (f $\left., \xi_{2}, \eta_{a}, \mathscr{g}\right), 1 \leq \mathrm{a} \leq \mathrm{s}$, with complemented frames, cf. [4]. A submanifold $\mathrm{j}: \mathrm{N} \rightarrow \mathrm{M}^{2 \mathrm{n}+\mathrm{s}}$ is said to be a framed C.R. submanifold if it is tangent to each structure vector $\xi$ of $M^{2 n+s}$ and it carries a pair of complementary (with respect to $\mathrm{G}=\mathrm{j}^{*} \mathscr{C}$ ) smooth distributions $\mathscr{D}, \mathscr{D}^{\perp}$ such that $\mathrm{f}_{\mathrm{x}}\left(\mathscr{D}_{\mathrm{x}}\right) \subseteq \mathscr{D}_{\mathrm{x}}$, $f_{x}\left(\mathscr{D}_{x}^{\perp}\right) \subseteq T_{x}(N) \perp$, for all $x \in N$, where $T(N)^{\perp} \rightarrow N$ stands for the normal bundle of j. Cf. I.MIHAI, [5], L.ORNEA, [6]. Since f-structures are known to generalize both almost complex $(s=0)$ structures and almost contact $(s=1)$ structures, the notion of framed C.R. submanifold containes those of a C.R. submanifold (see e.g. [7], p.83) of an almost Hermitian manifold and of a 
contact C.R. submanifold (see e.g. [7], p.48) of an almost contact metrical manifold.

Let $\bar{\pi}: \mathrm{H}^{2 \mathrm{n}+\mathrm{s}} \rightarrow \mathbb{C P ^ { \mathrm { n } }}$ be the generalized Hopf fibration, as given by D.E.BLAIR, [8]. Leaving definitions momentarily aside we may formulate the following:

\section{THEOREM A}

i) Let $\mathrm{N}$ be a framed C.R. submanifold of an $\mathscr{S}_{\text {-manifold }} \mathrm{M}^{2 \mathrm{n}+\mathrm{s}}$. Then the f-anti-invariant distribution $\mathscr{D}^{\perp}$ of $\mathrm{N}$ is completely integrable.

ii) Any framed C.R. submanifold of $\mathrm{H}^{2 \mathrm{n}+\mathrm{s}}$, (carrying the standard $\mathscr{P}_{- \text {structure) }}$ is either a C.R. submanifold (s even) or a contact C.R. submanifold (s odd). The converse holds.

iii) Let $\mathrm{N}$ be an f-invariant (i.e. $\mathscr{D}^{\perp}=(0)$ ) submanifold of $\mathrm{H}^{2 \mathrm{n}+\mathrm{s}}$. Then $\mathrm{N}$ is totally-geodesic if and only if it is an $\mathscr{P}$-manifold of constant f-sectional curvature $1-\frac{3}{4} \mathrm{~s}$.

iv) Any f-invariant submanifold of $\mathrm{H}^{2 \mathrm{n}+\mathrm{s}}$ having a parallel second fundamental form is totally-geodesic.

It is known that compact regular contact manifolds are $S^{1}$ - principal fibre bundles over symplectic manifolds, cf. W.M. BOOTHBY \& H.C.WANG, [9]. Eversince this (today classical) paper has been published, several "Boothby-Wang type" theorems have been established, cf. e.g. A.MORIMOTO, [10], for the case of normal almost contact manifolds, S.TANNO, [11], for contact manifolds in the non-compact case; more recently, we may cite a result of I.VAISMAN, [12], asserting that compact generalized Hopf manifolds with a regular Lee field may be fibred over Sasakian manifolds, etc.

There exists today a large literature, cf. K.YANO \& M.KON, [7], concerned with the study of the geometry (of the second fundamental form) of a C.R. submanifold of a Kaehlerian ambient space. In particular, following the method of Riemannian fibre bundles (such as introduced by H.B.LAWSON, [13], towards studying submanifolds of complex space-forms, and developed successively by Y.MAEDA, [14], M.OKUMURA, [15]), K.YANO \& M.KON, [16], have taken under study contact C.R. submanifolds of a Sasakian manifold $M^{2 n+1}$ (where $M^{2 n+1}$ is previously fibred over a Kaehlerian manifold $M^{2 n}$ ) which are themselves $S^{1}$-fibrations over C.R. submanifolds of $M^{2 n}$.

The last piece of the mosaic we are going to mend is the concept of canonical cohomology class (here after refered to as the Chen class) of a C.R. submanifold . Cf. B.Y.CHEN, [17], with any C.R. submanifold $M$ of a Kaehlerian manifold there may be associated a cohomology class $c(M) \in H^{2 p}(M ; \mathbb{R})$, where $p$ stands for the complex dimension of the holomorphic distribution of $M$. Although the canonical Hermitian structure (cf. [18]) of $\mathrm{H}^{2 \mathrm{n}+3}$ is never Kaehlerian (cf. [8], p.174) we show that the Chen class of a C.R. submanifold may be constructed as well and obtain the following :

THEOREM B

Let $\mathrm{j}: \mathrm{N} \rightarrow \mathrm{H}^{2 \mathrm{n}+\mathrm{s}}$ be a closed (i.e. compact, orientable) submanifold tangent

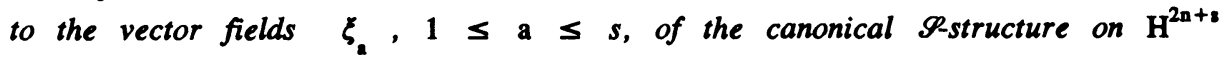
and assume there exists a $\mathrm{T}^{\mathrm{s}}$ - principal bundle $\pi: \mathrm{N} \rightarrow \mathrm{M}$ over a Cauchy- 
Riemann product $\left(\mathbf{M}, \mathscr{D}, \mathscr{D}^{\perp}\right), \quad \mathrm{i}: \mathbf{M} \rightarrow \mathbb{C P}^{\mathbf{n}},(\mathscr{D}$ is the holomorphic distribution), such that $\bar{\pi} \circ \mathrm{j}=\mathrm{i} \circ \pi$ and $\mathrm{j}$ is a diffeomorphism on fibres. If $\mathrm{s}$ is even then $\mathrm{N}$ is a C.R. submanifold whose totally-real foliation is normal to the characteristic field of $\mathrm{H}^{2 \mathrm{n}+\mathrm{s}}$ and whose Chen class $\mathrm{c}(\mathrm{N}) \in \mathrm{H}^{2 \mathrm{p}+\mathrm{s}}(\mathrm{N} ; \mathbb{R}), \mathrm{p}=\operatorname{dim}_{\mathbb{C}} \mathscr{D}$, is non-vanishing.

\section{2.- NOTATIONS, CONVENTIONS AND BASIC FORMULAE.}

Let $\mathrm{M}^{2 \mathrm{n}+\mathrm{s}}$ be a real $(2 \mathrm{n}+\mathrm{s})$-dimensional $\mathrm{C}^{\infty}$-differentiable connected manifold. Let $\underline{f}$ be an $f$-structure on $M^{2 n+s}$, i.e. a $(1,1)$-tensor field such that $\underline{\underline{t}}^{3}+\underline{\mathbf{f}}=0$ and $\operatorname{rank}(\underline{f})=2 n$ everywhere on $M^{2 n+s}$, cf. K.YANO, [19]. Assume that $\mathbf{f}$ has complemented frames, i.e. there exist the differential 1forms $\eta_{a}^{\prime}$ and the dual vector fields $\xi_{a}^{\prime}$ on $M^{2 n+s}$, i.e. $\eta_{a}^{\prime}\left(\xi_{b}^{\prime}\right)=\delta_{a b}, 1 \leq a, b \leq$ $s$, such that the following formulae hold:

$$
\eta_{a}^{\prime} \circ \underline{\mathbf{f}}=0, \quad \underline{\mathbf{f}}\left(\xi_{\mathbf{a}}^{\prime}\right)=0, \quad \underline{\mathbf{f}}^{2}=-\mathrm{I}+\eta_{\mathrm{a}}^{\prime} \otimes \xi^{\prime \mathbf{a}} .
$$

Throughout, one adopts the convention $\eta_{2}^{\prime}=\eta^{\prime 2}, \xi_{2}^{\prime}=\xi^{\prime 2}$.The $\mathrm{f}$ - structure is normal if $\left.[\mathrm{f}, \mathrm{f}]+\left(\mathrm{d} \eta^{\prime}\right) \otimes \xi^{\prime}\right)=0$, where [f, f] denotes the Nijenhuis torsion of $\underline{f}$, see e.g. H.NAKAGAWA, [20]. Let $\mathscr{C}$ be a compatible Riemaniann metric on $M^{2 n+s}$, i.e. one satisfying:

$$
\mathscr{E}(\underline{\mathrm{XX}}, \underline{\mathrm{fY}})=\mathscr{E}(\mathrm{X}, \mathrm{Y})-\eta_{\mathbf{z}}^{\prime}(\mathrm{X}) \eta^{\prime 2}(\mathrm{Y}) .
$$

Compatible metrics always exist, cf. D.E.BLAIR, [4]. Such (f, $\left.\xi_{1}^{\prime}, \eta_{1}^{\prime}, \mathscr{8}\right)$ has often been called a metrical f-structure with complemented frames. Let $\underline{F}(X, Y)=\mathscr{Q}(X, \underline{f})$ be its fundamental 2-form. Throughout we assume $M^{2 n+s}$ to be an $\mathscr{R}_{\text {manifold, }}$ cf. the terminology in [4], i.e. the given f-structure is

normal, its fundamental 2 -form is closed and there exist $s$ smooth real-valued functions $\alpha, \in \mathrm{C}^{\infty}\left(\mathrm{M}^{2 \mathrm{n}+\mathrm{s}}\right), 1 \leq \mathrm{a} \leq \mathrm{s}$, such that:

$$
\mathrm{d} \eta_{\mathrm{a}}^{\prime}=\alpha_{\mathrm{a}} \mathrm{F} \text {. }
$$

We shall need, cf. [4], [21], the following result. Let $M^{2 n+s}, n>1$, be a connected manifold carrying the $\mathscr{R}_{\text {structure }}\left(\mathrm{f}, \xi_{\dot{2}}^{\prime}, \eta_{2}^{\prime}, \mathscr{C}\right.$ ), $1 \leq \mathrm{a} \leq \mathrm{s}$. Then $\alpha_{2}$ are real constants, $\xi$, are Killing vector fields (with respect to $\mathscr{G}$ ) and the following relations hold:

$$
\underline{D}_{\mathrm{x}} \xi_{\mathbf{z}}=-\frac{1}{2} \alpha_{\mathbf{a}} \underline{\mathrm{f}} \mathrm{X}
$$

$$
\left(\mathrm{D}_{\mathrm{X}} \mathrm{f}\right) \mathrm{Y}=\frac{1}{2} \alpha^{\mathrm{a}}\left\{\left[\mathscr{E}(\mathrm{X}, \mathrm{Y})-\eta_{\mathrm{b}}^{\prime}(\mathrm{X}) \eta^{, \mathrm{b}}(\mathrm{Y})\right] \xi_{\mathrm{a}}^{\prime}-\left[\mathrm{X}-\eta_{\mathrm{b}}^{\prime}(\mathrm{X}) \xi^{, \mathrm{b}}\right] \eta_{\mathrm{a}}^{\prime}(\mathrm{Y})\right\}
$$

for any tangent vector fields $X, Y$ on $M^{2 n+s}$. Here $D$ denotes the Riemannian connection of $\left(\mathrm{M}^{2 \mathrm{n}+\mathrm{s}}, \mathscr{C}\right)$, and $\alpha^{2}=\alpha_{1}, 1 \leq \mathrm{a} \leq \mathrm{s}$.

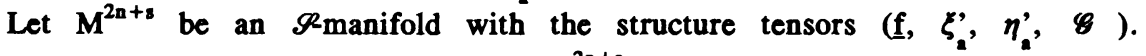
Let be the smooth s-distribution on $M^{2 n+8}$ spanned by $\xi_{1}, 1 \leq a \leq s$. By normality one has $\left[\xi_{a}^{\prime}, \xi_{b}^{\prime}\right]=0$, i.e. is involutive. If both and the structure vector fields $\xi$, are regular (in the sense of R.PALAIS, [22])

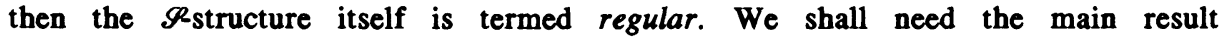
of D.E. BLAIR \& G.D.LUDDEN \& K.YANO, ([21], p.175). That is, let $\mathrm{M}^{2 \mathrm{n}+\mathrm{s}}$ be a

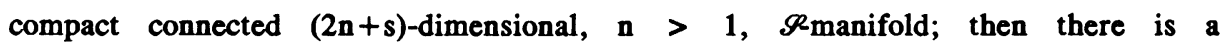
$T^{s}$-principal fibre bundle $\bar{\pi}: M^{2 n+8} \rightarrow M^{2 n}=M^{2 n+s} /$ and $M^{2 n}$ is a Kaehlerian 
manifold. Here $\mathrm{M}^{2 \mathrm{n}}$ denotes the leaf space of the s-dimensional foliation and $\mathrm{T}^{s}$ is the s-torus. Also, cf. ([21], p.178), $\gamma=\left(\eta_{1}^{\prime}, \ldots, \eta_{\mathrm{s}}^{\prime}\right)$ is a connection 1-form in $M^{2 n+s}\left(M^{2 n}, \bar{\pi}, T^{s}\right)$. If $X$ is a tangent vector field on $M^{2 n}$, let $X^{H}$ denote its horizontal lift with respect to $\gamma$. The Kaehlerian structure $(J, g)$ of $\mathrm{M}^{2 \mathrm{n}}$ is expressed by:

$$
\begin{aligned}
\mathrm{J} \mathbf{X} & =\bar{\pi}_{*} \underline{\mathbf{f}} \mathrm{X}^{\mathrm{H}} \\
\overline{\mathbf{g}}(\mathrm{X}, \mathrm{Y}) & =\mathscr{G}\left(\mathrm{X}^{\mathrm{H}}, \mathrm{Y}^{\mathbf{H}}\right) .
\end{aligned}
$$

Let $\mathscr{L}$ be the smooth $2 \mathrm{n}$-distribution on $\mathrm{M}^{2 \mathrm{n}+\mathrm{s}}$ defined by the Pfaffian equations $\eta_{2}^{\prime}=0,1 \leq \mathrm{a} \leq \mathrm{s}$. Then $\mathscr{L}$ is precisely the horizontal distribution of $\gamma$. Since $\eta^{\prime} \circ \underline{\mathrm{f}}=0$, the f-structure preserves the horizontal distribution. Therefore (2.6) may be also written $(\mathrm{J} \mathrm{X})^{\mathrm{H}}=\underline{\mathrm{f}} \mathrm{X}^{\mathrm{H}}$. Let $\bar{\nabla}$ be the Riemannian connection of $\left(\mathrm{M}^{2 \mathrm{n}}, \overline{\mathrm{g}}\right)$. By $([21], \mathrm{p} .179)$ one has:

$$
\underline{\mathrm{D}}_{\mathrm{X}^{\mathrm{H}}} \mathrm{Y}^{\mathrm{H}}=\left(\nabla \mathrm{X}^{\mathrm{Y}}\right)^{\mathrm{H}}+-_{-}^{1} \alpha^{*} \mathscr{C}\left(\underline{\mathrm{f}} \mathrm{X}^{\mathrm{H}}, \mathrm{Y}^{\mathrm{H}}\right) \xi^{\prime} .
$$

\section{REMARK}

Let $\pi: N \rightarrow M$ be a Riemannian submersion, cf. B.O'NEILL, [23]. Then $\operatorname{Ker}\left(\pi_{*}\right)$ is the vertical distribution, while its complement (with respect to the Riemannian metric of $\mathrm{N}$ ) is the horizontal distribution of the Riemannian submersion. As to our $\bar{\pi}: \mathbf{M}^{2 n+8} \rightarrow M^{2 n}$ a number of important coincidences occur. Firstly, if $M^{2 n}$ is assigned the Riemannian metric (2.7), then $M^{2 n+s} \rightarrow M^{2 n}$ is a Riemannian submersion. Moreover $=\operatorname{Ker}\left(\bar{\pi}_{*}\right)$ and therefore the horizontal distribution of the Riemannian submersion is precisely $\mathscr{L}$.

Let $N$ be an $(m+s)$-dimensional submanifold of $M^{2 n+s}$, and $M$ an m-dimensional submanifold of $M^{2 n}$, such that there exists a fibering $\pi: N \rightarrow M$ such that $\bar{\pi} \circ \mathrm{j}=\mathrm{i} \circ \pi$ and $\mathrm{j}$ is a diffeomorphism on fibres. Both $\mathrm{i}: \mathbf{M} \rightarrow \mathbf{M}^{2 n}$, $\mathrm{j}: \mathrm{N} \rightarrow \mathrm{M}^{2 \mathrm{n}+\mathrm{s}}$ stand for canonical inclusions. Let $\mathrm{g}=\mathrm{i}^{*} \overline{\mathrm{g}}, \mathrm{G}=\mathrm{j}^{*} \mathscr{G}$ be the induced metrics on $M$ and $N$, respectively. Also we denote by $\nabla, D$ the corresponding Riemannian connections of $(M, g)$ and $(N, G)$, respectively. Let $B$ (resp. h) be the second fundamental form of $i$ (resp. j) and denote by $A$ (resp. W) the Weingarten forms. Let $T(M) \stackrel{\perp}{\rightarrow} M$ (resp. $T(N){ }^{\perp} \rightarrow N$ ) be the normal bundle of $\mathrm{i}$ (resp. j). We put $\xi_{a}=\tan \left(\xi_{a}^{\prime}\right), \xi_{a}^{\perp}=$ nor $\left(\xi_{a}^{\prime}\right)$, where $\tan { }_{x}$, nor stand for the projections associated with the direct sum decomposition $T_{x}\left(M^{2 n^{x}+s}\right)=$ $T_{x}(N) \oplus T_{x}(N) \perp, x \in N$. Then the Gauss and Weingarten formulae, (cf. e.g. [24],p.39-40), of $i, j$ and our (2.8) lead to:

$$
\begin{aligned}
& \mathrm{D}_{\mathrm{X}^{\mathrm{H}}} \mathrm{Y}^{\mathrm{H}}=\left(\nabla_{\mathrm{X}} \mathrm{Y}\right)^{\mathrm{H}}+{ }_{-\frac{1}{2}} \alpha^{\mathrm{A}} \mathscr{E}\left(\mathbf{f} \mathrm{X}^{\mathrm{H}}, \mathrm{Y}^{\mathrm{H}}\right) \xi \\
& h\left(X^{H}, Y^{H}\right)=B(X, Y)^{H}+\frac{1}{2} \alpha^{\mathbf{A}} \mathscr{E}\left(f X^{H}, Y^{H}\right) \xi \text {. } \\
& \mathrm{W}_{\mathrm{V}^{\mathrm{H}}} \mathrm{Y}^{\mathrm{H}}=\left(\mathrm{A}_{\mathrm{V}} \mathrm{X}\right)^{\mathrm{H}}-\frac{1}{2} \alpha^{\mathrm{a}} \mathscr{E}\left(\mathrm{f} \mathrm{X}^{\mathrm{H}}, \mathrm{V}^{\mathrm{H}}\right) \xi_{\mathrm{a}} \\
& \mathrm{D}^{\perp} \mathrm{X}^{\mathrm{H}} \mathrm{V}^{\mathrm{H}}=\left(\nabla \frac{1}{\mathrm{X}} \mathrm{V}\right){ }^{\mathrm{H}}+-_{2}^{1} \alpha^{2} \mathscr{E}\left(\underline{\mathrm{f}} \mathrm{X}^{\mathrm{H}}, \mathrm{V}^{\mathrm{H}}\right) \xi^{\perp}
\end{aligned}
$$

for any tangent vector fields $X, Y$ on $M$, respectively any cross-section $V$ in $\mathrm{T}(\mathrm{M})^{\perp} \rightarrow \mathrm{M}$. Here $\nabla^{\perp}, \mathrm{D}^{\perp}$ stand for the normal connections of $i, j$. Of course, towards obtaining our (2.9) - (2.12) one exploits the fact that $\left(i_{*} X\right)^{\mathbf{H}}$ is tangent to $N$, while $V^{H}$ is a cross-section in $T(N) \stackrel{\perp}{\rightarrow} N$. 


\section{REMARKS}

1) Let $\mathrm{H}(\mathrm{i})=\frac{1}{\mathrm{~m}} \operatorname{Trace}(\mathrm{B}) \quad\left(\right.$ resp. $\mathrm{H}(\mathrm{j})=\frac{1}{\mathrm{~m}+\mathrm{s}} \operatorname{Trace}(\mathrm{h})$ ) be the mean curvature vector of $\mathrm{i}$ (resp. $\mathrm{j}$ ). As an application of our (2.9) - (2.12) one may derive:

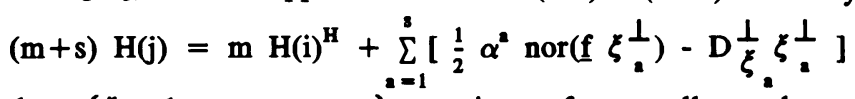

provided that $\left\{\xi_{a}: 1 \leq a \leq s\right\}^{2=1}$ consists of mutually orthogonal unit vector fields. In particular, if $\mathrm{N}$ is tangent to each structure vector $\xi, 1 \leq a \leq$ $\mathrm{s}$, then $\mathrm{N}$ is minimal if and only if $\mathrm{M}$ is minimal. Indeed, if $\mathrm{X}$ is tangent to $\mathrm{N}$, then (2.4) and the Gauss - Weingarten formulae lead to:

$$
\begin{gathered}
\mathrm{D}_{\mathrm{X}} \xi_{2}=\mathrm{W}_{\xi} \perp \mathrm{X}-\frac{1}{2} \alpha_{2} \tan (\mathrm{f} \mathrm{X}) \\
\mathrm{h}\left(\mathrm{X}, \xi_{2}\right)+\mathrm{D}_{\mathrm{X}}^{\perp} \xi_{2}^{\perp}=-\frac{1}{2} \alpha_{2} \text { nor (f X). }
\end{gathered}
$$

Now, if $\left\{\xi_{i}: 1 \leq a \leq s\right\}$ are orthonormal, one uses a frame $\left\{X_{i}, \xi_{a}^{H}\right\}$ (where $\left\{X_{i}\right.$ : $1 \leq \mathrm{i} \leq \mathrm{m}\}$ is an orthonormal tangential frame of $M$ ) such as to compute $H(j)$.

2) Generally, if $N$ is a submanifold of the $\mathscr{R}_{\text {manifold }} M^{2 n+s}$ and $N$ is normal to some $\xi_{1}^{\prime}$ with $\alpha_{2}=0$ then tangent spaces at points of $\mathrm{N}$ are f-anti-invariant, i.e. $\underline{f}_{x}\left(T_{x}(N)\right) \subseteq T_{x}(N) \perp, x \in N$. Indeed, by (2.4) and the Weingarten formula of $N$ in $M^{2 n+s}$, one has $\mathscr{E}(\alpha, f X, Y)=-2 \mathscr{G}\left(D_{X} \xi_{a}, Y\right)=2 \mathscr{E}\left(W_{\xi} \perp X, Y\right)$ where from $W_{\xi} \perp=0$ and $\underline{f} X$ is normal to $N$.

\section{3. $\mathscr{R}$ MANIFOLDS AS HERMITIAN OR NORMAL ALMOST CONTACT}

\section{METRICAL MANIFOLDS.}

We denote by $C P^{n}$ the complex projective space with constant holomorphic sectional curvature 1 (with Fubini - Study metric) and complex dimension n, and by $\mathrm{S}^{2 n+1}$ the $(2 \mathrm{n}+1)$-dimensional unit sphere carrying the standard Sasakian structure. Let $\pi^{1}: S^{2 n+1} \rightarrow C P^{n}$ be the Hopf fibration and set $H^{2 n+s}=\left\{\left(p_{1}, \ldots, p_{s}\right) \in S^{2 n+1} \times \ldots \times S^{2 n+1} \mid \pi^{1}\left(p_{1}\right)=\ldots=\pi^{1}\left(p_{s}\right)\right\}$.

We define a principal toroidal bundle by the commutative diagram:

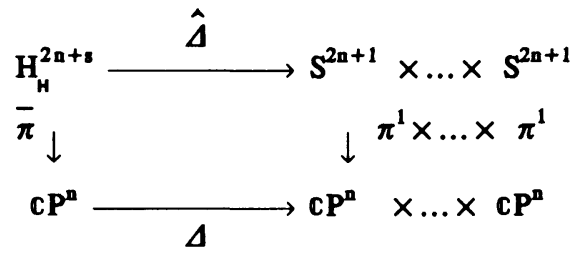

where $\Delta$ denotes the diagonal map, while $\hat{\Delta}$ stands for the canonical inclusion. Let $\eta^{\prime}$ be the standard contact 1 -form on $s^{2 n+1}$. We put $\eta_{a}^{\prime}=\hat{\Delta}^{*} \Delta^{*} \eta^{\prime}, 1$ $\leq a \leq s$ where $\Delta_{a}: S^{2 n+1} \times x S^{2 n+1} \rightarrow S^{2 n+1}$ are natural projections. Let $\Omega$ be the Kaehler 2-form of $\mathbf{C P}^{\mathrm{n}}$. Then on one hand $\gamma=\left(\eta_{1}^{\prime}, \ldots, \eta_{z}^{\prime}\right)$ is a connection 1-form in $\mathrm{H}^{2 \mathrm{n}+\mathrm{s}}\left(\mathrm{CP}^{\mathrm{n}}, \bar{\pi}, \mathrm{T}^{3}\right)$, and on the other $\mathrm{d} \eta_{\Omega}^{\prime}=\bar{\pi}^{*} \Omega$, such that one may apply theorem 3.1 of [8], (p.163) such as to yield a natural $\mathscr{R}_{\text {structure on }}$ $\mathrm{H}^{2 \mathrm{n}+\mathrm{s}}$. (Cf also [4], p.173). Let (f, $\xi_{a}, \eta_{2}, \mathscr{C}$ ) be the canonical $\mathscr{R}_{\text {structure }}$ 
of $\mathrm{H}^{2 \mathrm{n}+\mathrm{s}}$. If $\mathrm{S}$ is even one sets:

$$
\mathscr{L}=\underline{\mathbf{f}}+\sum_{\mathrm{i}=1}^{\mathrm{s}}\left\{\eta_{\mathrm{i}} \otimes \boldsymbol{\xi}_{\mathrm{i}} * \boldsymbol{\eta}_{\mathrm{i}} \otimes \boldsymbol{\xi}_{\mathrm{i}}\right\}
$$

where $\mathrm{i} \cdot=\mathrm{i}+\frac{\mathrm{s}}{2}, 1 \leq \mathrm{i} \leq \frac{\mathrm{s}}{2}$. If $\mathrm{s}$ is odd, one labels the 1-forms $\eta_{\mathrm{a}}$ as follows: $\eta_{0}, \eta_{\mathrm{i}}, \eta_{\mathrm{i} *}, \mathrm{i} * \mathrm{i}+\mathrm{r}, 1 \leq \mathrm{i} \leq \mathrm{r}, \mathrm{s}=2 \mathrm{r}+1$, and similarly for the tangent vector fields $\xi_{i}$. We consider:

$$
\left.\varphi=\underline{\mathbf{f}}+\sum_{\mathrm{i}=1}^{\mathrm{r}} \eta_{\mathrm{i}} \otimes \boldsymbol{\xi}_{\mathrm{i}} \cdot-\eta_{\mathrm{i}} \cdot \otimes \boldsymbol{\xi}_{\mathrm{i}}\right\}
$$

The characteristic 1-form of $\mathrm{H}^{2 \mathrm{n}+\mathrm{s}}$, $\mathrm{s}$ even, is defined by:

$$
\omega=2 \sum_{i=1}^{8 / 2}\left\{\eta_{i}-\eta_{i} .\right\}
$$

Let $\mathrm{B}=\omega^{\dagger}$ be the characteristic field, where + means raising of indices by $\mathscr{G}$. REMARKS

1) If $s$ is even then $\left(\mathrm{H}^{2 \mathrm{n}+\mathrm{s}}, \mathscr{\mathscr { S }}, \mathscr{G}\right)$ is a Hermitian non-Kaehlerian manifold and its characteristic form is parallel. Indeed, if $\mathrm{s}$ is even, then $\mathscr{\&}$ given by (3.1) is a complex structure and $\left(\mathrm{H}^{2 \mathrm{n}+s}, \mathscr{\&}, \mathscr{C}\right)$ turns to be a Hermitian manifold, (cf. prop.4.1 in [8], p.174). Let $\tilde{F}(X, Y)=\mathscr{E}(\mathrm{X}, \mathscr{\&} \mathrm{Y})$ be its Kaehler 2-form. By (3.1) it follows that $\tilde{F}=F-2 \sum_{i=1}^{s / 2} \eta_{i} \wedge \eta_{i}$; consequently (3.3) leads to

$$
\mathrm{dF}=\omega \wedge \underline{\mathbf{F}}
$$

i.e. $\mathscr{C}$ is not a Kaehler metric. Now our (2.4) yields $\underline{D} \omega=\frac{1}{2} \sum_{i=1}^{8 / 2}\left(\alpha_{i}-\bar{\alpha}_{i}\right.$ ) F

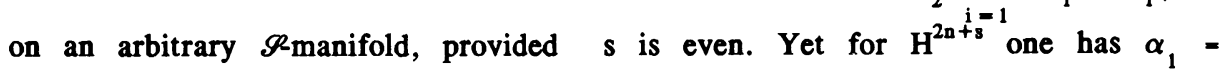
$\ldots=\alpha_{\mathrm{z}}$, (cf.[8],p.173), i.e. $\omega$ is parallel.

2) Since $\mathrm{d} \eta^{\prime}=\bar{\pi}^{*} \Omega, 1 \leq \mathrm{a} \leq \mathrm{s}$, it follows that $\omega$ is closed. Therefore $\mathrm{H}^{2 \mathrm{n}+\mathrm{s}}$, $\mathrm{S}$ even, admits the canonical foliation $\mathscr{F}$ defined by the Pfaffian

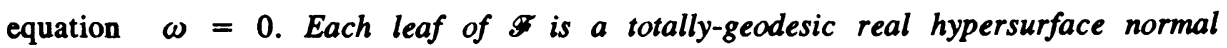
to the characteristic field of $\mathrm{H}^{2 \mathrm{n}+\mathrm{s}}$.

3) Consider the submanifolds $i: M \rightarrow C P^{n}$ and $j: N \rightarrow H^{2 n+8}$ and assume that a $T^{s}$-subbundle $\pi: N \rightarrow M$ of the generalized Hopf fibration, i.e. $\bar{\pi} \circ j=i \circ \pi$ and $j$ is a diffeomorphism on fibres. Suppose $N$ is tangent to the structure vectors $\xi$ of the $\mathscr{R}_{\text {manifold }} \mathrm{H}^{2 \mathrm{n}+3}$. Then $\mathrm{M}$ is a C.R. submanifold of $\mathrm{CP}^{\mathrm{n}}$ if and only if $\mathrm{N}$ is either a C.R. submanifold of $\left(\mathrm{H}^{2 \mathrm{n}+\mathrm{s}}, \mathscr{Z}, \mathscr{C}\right)$ or a contact C.R. submanifold of $\left(\mathrm{H}^{2 \mathrm{n}+\mathrm{s}}, \varphi, \xi_{0}, \eta_{0}, \mathscr{C}\right)$. Note firstly that, if $\mathrm{s}$ is odd, then $\left(\varphi, \xi_{0}, \eta_{0}, \mathscr{G}\right)$ is a normal almost contact metrical (a. ct. m.) structure on $\mathrm{H}^{2 \mathrm{n}+\mathrm{s}}$, (cf. [8], p.175). If $\xi_{a}^{\perp}=0,1 \leq \mathrm{a} \leq \mathrm{s}$, and $\mathrm{s}$ is even then:

$$
\mathscr{E} \xi_{\mathrm{i}}=\xi_{\mathrm{i}^{*}}, \quad \mathscr{E} \xi_{\mathrm{i}^{*}}=-\xi_{\mathrm{i}}, \quad \mathscr{D} \mathrm{X}^{\mathrm{H}}=(\mathrm{J} \mathrm{X})^{\mathrm{H}}
$$

for any tangent vector field $X$ on $M$, cf.(2.6). Let us define $\mathscr{P} Y=\tan (\mathscr{S} Y)$, $\mathscr{P} \mathrm{Y}=$ nor $(\mathscr{Q} \mathrm{Y})$, for any tangent vector field $\mathrm{Y}$ on $\mathrm{N}$. Then:

$$
\mathscr{P} \mathscr{P} \xi_{\mathrm{i}}=0, \quad \mathscr{P} \mathscr{P} \xi_{\mathrm{i}}{ }^{*}=0, \quad \mathscr{P} \mathscr{P} \mathrm{X}^{\mathrm{H}}=(\mathrm{F} \mathrm{P} \mathrm{X})^{\mathrm{H}}
$$

where F, $P$ are defined by (1.1) in [7] (p.76). Suppose for instance that (M, $\left.\mathscr{D}, \mathscr{D}^{\perp}\right)$ is a C.R. submanifold of $C P^{\mathrm{n}}$. Then $P$ is $\mathscr{D}$-valued, while $\mathrm{F}$ vanishes on 
$\mathscr{D}$, i.e. FP $=0$. By (3.6) one has $\mathscr{P} \mathscr{P}=0$, and thus one may apply theor. 3.1 in [7] (p.87), such as to conclude that $\mathrm{N}$ is a C.R. submanifold of $\left(\mathrm{H}^{2 \mathrm{n}+8}, \mathscr{Z}\right.$, $\mathscr{C}$ ). Note that, although stated for submanifolds in Kachlerian manifolds, theor.3.1 of [7] (p.87) actually holds for the general case of an arbitrary almost Hermitian ambient space. The case $s$ odd follows similarly from theor. 2.1 of [7] (p.55) which may be easily refined from the Sasakian case to the general case of a. ct. m. structures.

4) Let $\left(M, \mathscr{D}, \mathscr{D}^{\perp}\right)$ be a C.R. submanifold of $C P^{\mathrm{n}}$, where $\mathscr{D}$ (resp. $\mathscr{D}^{\perp}$ ) denotes the holomorphic (resp. totally-real) distribution. Let $\pi: \mathbf{N} \rightarrow \mathbf{M}$ be a $\mathrm{T}^{s}$-bundle as in Remark 3). Let $\mathscr{D}_{\mathrm{N}}, \mathscr{D} \frac{1}{\mathrm{~N}}$ be the holomorphic and totally-real (resp. the $\varphi$-invariant and $\varphi$-anti- invariant) distributions of $N$, provided that $s$ is even (resp. $s$ is odd). Let $L_{\mathrm{N}, \mathrm{x}}$ the natural projection on the first term of the direct sum decomposition $T_{x}^{N}(N)=\mathscr{D}_{N, x} \oplus \mathscr{D}_{N}^{\perp}, x \in N$. Cf. (3.7) in [7] (p.86), (resp. cf. (2.10) in [7] (p.53)) if $\mathrm{s}$ is even (resp. if $\mathrm{s}$ is odd) then ${ }_{\mathrm{N}}$ is expessed by $l_{\mathrm{N}}=-\mathscr{F}^{2}$ (resp. by $\left.l_{\mathrm{N}}=-\mathscr{P}^{2}+\eta_{0} \otimes \xi_{0}\right)$ where $\mathscr{F} \mathrm{Y}=\tan (\mathscr{S} \mathrm{Y})$, (resp. $\mathscr{P} \mathrm{Y}=\tan (\varphi \mathrm{Y})$ ). In both cases one has:

$$
l_{\mathrm{N}} \xi_{\mathrm{a}}=\xi_{\mathrm{a}}, 1 \leq \mathrm{a} \leq \mathrm{s}, \quad l_{\mathrm{N}} \mathrm{X}^{\mathrm{H}}=(\ell \mathrm{X})^{\mathrm{H}}
$$

where $l=-P^{2}$. As the sum $\mathscr{D}_{\mathrm{x}}^{\mathrm{H}}+M_{\mathrm{x}}, \mathrm{x} \in \mathrm{N}$, is direct one obtaines $\mathscr{D}_{\mathrm{N}, \mathrm{x}}=\mathscr{Q}_{\mathrm{x}}^{\mathrm{H}} \oplus$ $m_{x}, x \in N$. Indeed, one inclusion follows from (3.7). Conversely, let $X^{\prime} \in \mathscr{D}_{N}$, then $X^{\prime}=(\iota X)^{H}+\left(\zeta^{\perp} X\right)^{H}+\lambda^{2} \xi_{2}, \lambda^{2} \in C^{\infty}(N), \iota^{\perp}=I-\ell$. By applying $\iota_{N}^{N}$ to both members one proves $X^{\prime} \in \mathscr{D}^{\mathrm{H}} \oplus$. It is also straightforward that $\left(\mathscr{D}^{\perp}\right)^{\mathrm{H}}=$ $\Phi \frac{1}{N}$.

\section{4.- FRAMED CAUCHY-RIEMANN SUBMANIFOLDS}

S. GOLDBERG, [25], has inaugurated a program of unifying the treatment of the cases $s$ even, and $s$ odd, and studied f-invariant submanifolds of codi-

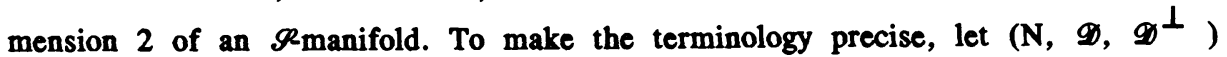
be a framed C.R. submanifold of $M^{2 n+s}$; we call $N$ an f-invariant (resp. f-anti-invariant) submanifold if $\mathscr{D}_{x}^{\perp}=(0)$, (resp. if $\mathscr{D}_{x}=(0)$ ), for any $x \in N$.

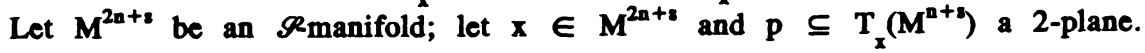
(Cf.[8], p.159), $p$ is an $f$-section if it is spanned by $\left\{X, f X_{x}\right\}$ for some unit tangent vector $\mathrm{X} \in \mathscr{\mathscr { L }}_{\mathrm{x}}$. The Riemannian sectional curvature of $\left(\mathrm{M}^{2 \mathrm{n}+3}, \mathscr{C}\right)$ restricted to f-sections is refered to as the f-sectional curvature of the

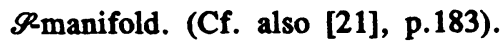

At this point we may establish i) of theor. A. Let $X, V$ be respectively a tangent vector field on $N$ and a cross-section in $T(N) \perp \rightarrow N$. We set $P X=$ $\tan (\mathbf{f} X), \quad F X=\operatorname{nor}(\underline{f} V)$ and $f V=\operatorname{nor}(f) V)$. The following identities hold as direct consequences of definitions:

$$
\begin{aligned}
& P^{2}+t F=-I+\eta \otimes \xi^{*}, \quad F P+f F=0, \quad P t+t f=0, \\
& \mathrm{~F} t+\mathrm{f}^{2}=-\mathrm{I}, \quad \mathrm{f} l=\mathrm{P} \ell, \quad \mathrm{F} \ell=0 \text {, }
\end{aligned}
$$

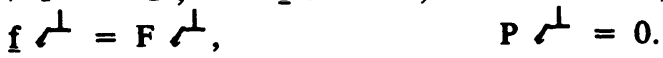

Using (2.5) and the Gauss - Weingarten formulae of $N$ in $M^{2 n+8}$ one obtaines:

$\left(D_{X} P\right) \mathbf{Y}=\mathbf{W}_{\text {FY }} \mathbf{X}+\mathrm{t} \mathbf{h}(\mathbf{X}, \mathbf{Y})+$

$$
+\frac{1}{2} \alpha^{a}\left\{\left[\mathrm{G}(\mathrm{X}, \mathrm{Y})-\eta_{b}(\mathrm{X}) \eta^{b}(\mathrm{Y})\right] \xi_{\mathrm{a}}-\left[\mathrm{X}-\eta_{\mathrm{b}}(\mathrm{X}) \xi^{\mathrm{b}}\right] \eta_{\mathrm{a}}(\mathrm{Y})\right\}
$$

for any tangent vector fields $X, Y$ on $N$. Let $X, Y \in \mathscr{D}^{\perp}$. As $D$ is torsion-free 
and by (4.2) one obtains:

$$
\mathrm{P}[\mathrm{X}, \mathrm{Y}]=\mathrm{W}_{\mathrm{FX}} \mathrm{Y}-\mathrm{W}_{\mathrm{FY}} \mathrm{X}+\alpha^{\mathrm{a}}\left\{\frac{1}{2}(\mathrm{X} \wedge \mathrm{Y}) \xi_{\mathrm{a}}+\left(\eta_{\mathrm{a}} \wedge \eta_{\mathrm{b}}\right)(\mathrm{X}, \mathrm{Y}) \xi^{\mathrm{b}}\right\} \text { (4.3) }
$$

At this point we may establish the following:

\section{LEMMA}

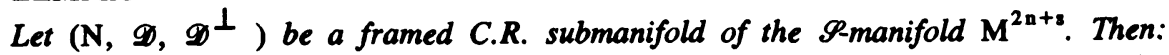

$\mathrm{W}_{\mathrm{FX}} \mathrm{Y}=\mathrm{W}_{\mathrm{FY}} \mathrm{X}+\frac{1}{2} \alpha^{2}\left\{\eta_{\mathrm{a}}(\mathrm{X}) \mathrm{Y}-\eta_{2}(\mathrm{Y}) \mathrm{X}-\left[\eta_{2}(\mathrm{X}) \eta_{2}(\mathrm{Y})-\eta_{2}(\mathrm{Y}) \eta_{2}(\mathrm{X})\right] \xi^{\mathrm{b}}\right\}$

for any $\mathrm{X}, \mathrm{Y} \in \mathscr{D}^{\perp}$.

Proof. By (4.1), $P$ vanishes on $\mathscr{D}^{\perp}$. Using (4.2), for any $X, Y \in \mathscr{D}^{\perp}, Z \in T(N)$, one has:

$$
\begin{gathered}
0=\mathrm{G}\left(\left(\mathrm{D}_{\mathrm{Z}} \mathrm{P}\right) \mathrm{X}, \mathrm{Y}\right)=\mathrm{G}\left(\mathrm{W}_{\mathrm{FX}} \mathrm{Z}, \mathrm{Y}\right)+\mathrm{G}(\mathrm{t} \mathrm{h}(\mathrm{Z}, \mathrm{X}), \mathrm{Y})+ \\
+\frac{1}{2} \alpha^{2}\left\{\mathrm{G}(\mathrm{Z}, \mathrm{X}) \eta_{\mathrm{a}}(\mathrm{Y})-\mathrm{G}(\mathrm{Z}, \mathrm{Y}) \eta_{\mathrm{a}}(\mathrm{X})+\left[\eta_{\mathrm{a}}(\mathrm{X}) \eta^{\mathrm{b}}(\mathrm{Y})-\eta_{\mathrm{a}}(\mathrm{Y}) \eta^{\mathrm{b}}(\mathrm{X})\right] \eta_{\mathrm{b}}(\mathrm{Z})\right\}
\end{gathered}
$$

and finally $G(t h(Z, X), Y)=-G\left(W_{F Y} X, Z\right)$ leads to (4.4).

By (4.3) and the above lemma we conclude $P[X, Y]=0$, i.e. $D^{\perp}$ is involutive.

Let us prove now ii) in theor. A. We analyse for instance the case $s$ even. Let $\mathrm{N}$ a framed C.R. submanifold of $\mathrm{H}^{2 \mathrm{n}+3}$. Let

$$
\left.\mathscr{P}=\mathrm{P}+\sum_{\mathrm{i}=1}^{8 / 2} \eta_{\mathrm{i}} \otimes \xi_{\mathrm{i}^{*}}-\eta_{\mathrm{i}^{*}} \otimes \xi_{\mathrm{i}}\right\}, \quad \mathscr{P}=\mathrm{F}
$$

Next $\mathscr{S}=$ F P $=0$, and one applies theor.3.1 of [7], p.87. The case $s$ odd being similar is left as an exercise to the reader. To prove the converse of ii) in theor.A we need to characterize framed C.R. submanifolds as follows. Let $\mathrm{N}$ be a framed C.R. submanifold of an $\mathscr{R}_{\text {manifold }} \mathrm{M}^{2 n+8}$. Then (4.1) leads to $\mathrm{P} \ell=\mathrm{P}, \mathrm{F} \mathbf{P}=0$, f $\mathrm{F}=0$, etc. One obtaines the following statement. Let $\mathrm{N}$ be a submanifold of the $\mathscr{S}_{\text {-manifold }} \mathrm{M}^{2 \mathrm{n}+3}$ such that $\mathrm{N}$ is tangent to the structure vectors $\xi_{2}$. Then $\mathrm{N}$ is a framed C.R. submanifold of $\mathrm{M}^{2 \mathrm{n}+\mathrm{s}}$ if and only if $\mathrm{F} \mathrm{P}=$ 0 . We have proved the necessity already. Viceversa, let us put by definition / $=-P^{2}+\eta_{2} \otimes \zeta^{2}, \downarrow=I-l$. Since F P $=0$, the projections $\iota, \downarrow$ make $N$ into a framed C.R. submanifold, Q.E.D. Now the converse of ii) in theor. $A$ is easily seen to hold, i.e. both C.R. submanifolds of $\left(\mathrm{H}^{2 \mathrm{n}+3}, \mathscr{Z}, 8\right)$, s even, and contact C.R. submanifolds of $\left(\mathrm{H}^{2 \mathrm{n}+s}, \varphi, \xi_{0}, \eta_{0}, 8\right)$, s odd, are framed C.R. submanifolds.

REMARKS

1) Let $\left(\mathrm{N}, \mathscr{D}, \mathscr{D}^{\perp}\right)$ be a framed C.R. submanifold of $\mathrm{H}^{2 \mathrm{n}+\mathrm{s}}$. By (4.5) one obtains:

$$
\mathscr{P}^{2}=\mathbf{P}^{2}-\eta^{2} \odot \zeta^{2}
$$

Now the notion of framed C.R. submanifold appears to be essentially on old concept. For not only $\mathrm{N}$ becomes a C.R. submanifold of the Hermitian manifold $\mathrm{H}^{2 \mathrm{n}+3}$, if for instance $\mathrm{S}$ is even, but its holomorphic and totally-real distributions are precisely $\mathscr{D}, \mathscr{D}^{\perp}$. Indeed, by (4.6) one has $l_{\mathrm{N}}=$ l, Q.E.D.

2) Due to (3.4) there is a certain similarity between $\mathscr{R}_{\text {manifolds and locally }}$ conformal Kaehler manifolds, cf. P.LIBERMANN, [26]. See also [12]. For instance, we may use the ideas in [2] (cf. also theor. 3.4 of [7], p.89) to 
give an other proof of the integrability of the f-anti-invariant distribution of a framed C.R. submanifold. Indeed, let $N$ be a framed C.R. submanifold of $H^{2 n+3}$, s even. Let $X \in \mathscr{D}, Z, W \in \mathscr{D}^{\perp}$. By (3.4) one has $0==3(\mathrm{~d} \tilde{F})(X, Y, W)$ $=-G([Z, W], J X)$. Hence $[Z, W] \in \mathscr{D}^{\perp}$. Note that, although $N$ is C.R. in the usual sense one could not apply theor.3.4 or theor.4.1 of [7] (p.89-90) since $\mathrm{H}^{2 \mathrm{n}+\mathrm{s}}$ is neither locally conformal Kaehler nor Kaehler.

To establish iii) let $\mathrm{N}$ be an f-invariant submanifold of $\mathrm{H}^{2 \mathrm{n}+3}$. As a consequence of (2.5), for any tangent vector fields $\mathrm{X}, \mathrm{Y}$ on $\mathrm{N}$ one has:

$$
\begin{gathered}
\left(\mathrm{D}_{\mathrm{X}} \underline{\mathrm{f}}\right) \mathrm{Y}=\frac{1}{2} \alpha^{\mathrm{a}}\left\{\left[\mathrm{G}(\mathrm{X}, \mathrm{Y})-\eta_{\mathrm{b}}(\mathrm{X}) \eta^{\mathrm{b}}(\mathrm{Y})\right] \xi_{\mathrm{a}}-\left[\mathrm{X}-\eta_{\mathrm{b}}(\mathrm{X}) \xi^{\mathrm{b}}\right] \eta_{\mathrm{a}}(\mathrm{Y})\right\} \\
\mathrm{h}(\mathrm{X}, \underline{\mathrm{f}} \mathrm{Y})=\underline{\mathrm{f}} \mathrm{h}(\mathrm{X}, \mathrm{Y}) .
\end{gathered}
$$

Let $\mathbf{k}(X, Y)$ be the Riemannian sectional curvature of the 2-plane spanned by the orthonormal pair $\{X, Y\}$ on $N$; using the Gauss equation, i.e. equation (2.6) in [24], (p.45), and the notations in [4], (p.161), i.e. $H(X)=k(X$, $\mathrm{fX}), \mathrm{X} \in \mathscr{L}$, one obtains:

$$
1-\frac{3}{4} s=H(X)+2\|h(X, X)\|^{2}
$$

as $\mathrm{H}^{2 \mathrm{n}+\mathrm{s}}$ has constant f-sectional curvature, (cf.[8], p.173). By (2.15) and f-invariance one has $h\left(X, \xi_{2}\right)=-\frac{1}{2} \alpha_{a} \operatorname{nor}(f X)=0$; a standard argument based on (4.8) leads to the proof.

To prove iv) one uses $D h=0,(2.15)$ and f-invariance, i.e. one has $h\left(\left(D_{X} \xi_{a}, Y\right)=0\right.$. Thus $\alpha_{a} h(f X, Y)=0$, by (2.14). For some $\alpha_{a}=0$ one uses (4.7). Finally, apply once more $f$ and notice that $\eta_{2}$ vanish on normal vectors. Thus $\mathrm{h}=0$.

\section{REMARK}

Let $F$ be the canonical foliation of $\mathrm{H}^{2 \mathrm{n}+\mathrm{z}}$. Let $\mathrm{N}$ be a framed C.R. submanifold of $\mathrm{H}^{2 \mathrm{n}+3}$, as above. Then $\mathscr{D}^{\perp} \subseteq \mathscr{F}$, i.e. the totally-real foliation of $\mathrm{N}$ (regarded as a C.R. submanifold, $\mathrm{s}$ even) is normal to the characteristic field $2 \sum_{i=1}^{s / 2}\left(\xi_{i}-\xi_{i}\right)$ of $H^{2 n+s}$. Indeed, since $\xi_{i} \in \mathscr{D}^{\perp}$, the $\eta_{2}$ vanish on $\mathscr{D} !$ Thus $\omega \circ \triangleleft=0$.

\section{5.- THE CHEN CLASS OF A CAUCHY-RIEMANN SUBMANIFOLD.}

Let $M$ be a C.R. submanifold of $\mathbf{C P}^{\mathbf{n}}$. Let $\pi: N \rightarrow M$ be a $T^{3}$ - fibration, as in theor. B. Assume $S$ is even. Then $N$ is a C.R.submanifold of $H^{2 n+s}$ and its totally-real distribution is integrable. We shall need the following:

\section{LEMMA}

The holomorphic distribution of $N$ is minimal.

Proof.

Note that we may not use lemma 4. in [17] (p.169) since its proof makes essential use of the Kaehler property. Neither could one use corollary 2.3 of [27] (p.291), (although $\mathscr{D} \frac{1}{\mathrm{~N}} \subseteq \mathscr{F}$ ) since $(\mathscr{\&}, \mathscr{B})$ fails to be locally conformal Kaehler. Now (2.4) - (2.5), (3.1) lead to:

$$
\begin{aligned}
\left(\mathrm{D}_{\mathrm{X}} \mathscr{D}\right) \mathrm{Y}=\frac{1}{2}\left\{\left[\mathscr{C}(\mathrm{X}, \mathrm{Y})-\eta_{\mathrm{b}}(\mathrm{X}) \eta^{\mathrm{b}}(\mathrm{Y})\right] \xi-\right. \\
\left.-\left[\mathrm{X}-\eta_{\mathrm{b}}(\mathrm{X}) \xi^{\mathrm{b}}\right] \eta(\mathrm{Y})\right\}-\frac{1}{4}\{\underline{\mathrm{F}}(\mathrm{X}, \mathrm{Y}) \mathrm{B}+\omega(\mathrm{Y}) \underline{\mathrm{f}} \mathrm{X}\}
\end{aligned}
$$


where $\eta=\sum_{i=1}^{s} \eta_{2}, \xi=\eta^{\dagger}$. Let $\mathrm{X} \in \mathscr{D}_{\mathrm{N}}, \mathrm{Z} \in \mathscr{D}_{\mathrm{N}}^{\perp}$. Using (5.1) we have:

$\left(\mathrm{Z}, \underline{\mathrm{D}}_{\mathrm{X}} \mathrm{X}\right)=\mathscr{E}\left(\mathscr{Z} \mathrm{Z}, \mathscr{\&} \underline{\mathrm{D}}_{\mathrm{X}} \mathrm{X}\right)=\mathscr{E}\left(\mathscr{Z} \mathrm{Z}, \underline{\mathrm{D}}_{\mathrm{X}} \mathscr{\&} \mathrm{X}\right)=\mathscr{E}\left(\mathrm{W}_{\mathscr{D}} \mathrm{X}, \mathscr{\&} \mathrm{X}\right)$.

Thus: $\mathscr{C}\left(\mathrm{Z}, \underline{\mathrm{D}}_{\mathrm{X}} \mathrm{X}+\underline{\mathrm{D}}_{\mathscr{D} \mathrm{X}} \mathscr{\mathscr { D }} \mathrm{X}\right)=0$ and $\mathscr{D} \frac{1}{\mathrm{~N}}$ follows to be minimal. Let $\mathrm{p}=\operatorname{dim}_{\mathbb{C}} \mathscr{D}$. Let $\left\{\mathrm{X}_{\mathrm{A}}: 1 \leq \mathrm{A} \leq 2 \mathrm{p}\right\}$ be a real orthonormal frame of $\mathscr{D}$, where $\mathrm{X}_{\mathrm{i}+\mathrm{p}}=\mathscr{D} \mathrm{X}_{\mathrm{i}}$, $1 \leq \mathrm{i} \leq \mathrm{p}$. Then $\left\{\mathrm{X}_{\mathrm{A}}^{\mathrm{H}}, \xi_{\mathrm{a}}\right\}$ is an orthonormal frame of $\mathscr{D}_{\mathbf{N}}$. Let $\lambda^{A}, 1 \leq A \leq 2 \mathrm{p}$, be differential 1-forms on $N$ defined by $\lambda^{\wedge}\left(X_{B}\right)=\delta_{B}^{\wedge}, \lambda^{\wedge}(Y)=0$, for any $\mathrm{Y} \in \mathscr{D}_{\mathrm{N}}^{\perp}$. Let $\lambda==\lambda^{1} \wedge \ldots \wedge \lambda^{2 p} \wedge \eta^{1} \wedge \ldots \wedge \eta^{2}$. Then $\lambda$ is a globally defined $(2 p+s)$-form on $N$, as $\mathscr{D}_{N}$ is orientable. We leave it as an exercise to the reader to follow the ideas in [17] (p.170) and show that since $\mathscr{D}_{\mathrm{N}}$ is minimal and $\mathscr{D} \frac{1}{N}$ integrable the $(2 \mathrm{p}+\mathrm{s})$-form $\lambda$ is closed. Thus $\lambda$ determines a cohomology class $c(N)=[\lambda] \in \mathrm{H}^{2 p+z}(N ; R)$ refered to as the Chen class of $N$.

To prove theor. B suppose $M$ is a C.R. product, i.e. $M$ is locally a product of a complex submanifold and a totally-real submanifold of $C^{n}$, see e.g. [28], (p.63). Now C.R. products have an integrable holomorphic distribution and a minimal totally-real distribution. By (2.8), for any tangent vector fields $\mathrm{X}, \mathrm{Y}$ on $\mathrm{CP}^{\mathrm{n}}$ one has:

$$
\left[X^{H}, Y^{H}\right]=[X, Y]^{H}-\alpha^{\mathbf{Q}} E\left(X^{H}, Y^{H}\right) \xi
$$

Then (5.2) used for $X=X_{A}, Y=X_{B}$ leads to $\left[X_{A}^{H}, X_{B}^{H}\right] \in \mathscr{D}_{N}$. Next, as $\mathbf{X}_{\mathbf{A}}^{\mathbf{H}}=\mathbf{O}$ one has

$$
\mathscr{S}^{\perp}\left[\mathrm{X}_{\mathrm{A}}^{\mathrm{H}}, \xi_{\mathrm{a}}\right]=\left(\underline{\mathrm{D}}_{\xi_{\mathrm{a}}} \stackrel{\mathcal{L}}{\mathcal{L}} \mathrm{X}_{\mathrm{A}}^{\mathrm{H}}-\mathscr{S}_{\mathrm{A}}^{\perp} \underline{\mathrm{D}}_{\mathrm{X}}^{\mathrm{H}} \xi_{\mathrm{a}} .\right.
$$

We need the following :

\section{LEMMA}

The covariant derivative $\left(\mathrm{D}_{\mathrm{X}} \mathscr{F}^{\mathrm{L}}\right) \mathrm{Y}=\mathrm{D}_{\mathrm{X}}^{1} \mathscr{F}^{\perp} \mathrm{Y}-\mathscr{F}^{\perp} \mathrm{D}_{\mathrm{X}} \mathrm{Y}$ of $\mathscr{F}^{\perp}$ is expressed by:

$$
\left(D_{X} \mathscr{P}^{ل}\right) \mathrm{Y}=-\mathrm{h}(\mathrm{X}, \mathscr{P} \mathrm{Y}) \tilde{\mathrm{f} h}(\mathrm{X}, \mathrm{Y})-\frac{1}{4} \omega(\mathrm{Y}) \mathrm{F} \mathrm{X}
$$

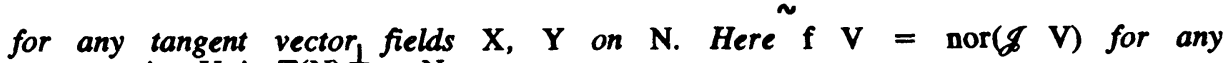
cross-section $\mathrm{V}$ in $\mathrm{T}(\mathrm{N}) \stackrel{ }{\rightarrow} \mathrm{N}$.

Proof.

Let also $\mathrm{t} V=\tan (\mathcal{V} \mathrm{V})$. Using the Gauss and Weingarten formulae of $\mathrm{N}$ in $\mathrm{H}^{2 \mathrm{n}+\mathrm{s}}$ one has:

$$
\begin{aligned}
& \left(\underline{D}_{X} \mathscr{D}\right)=\left(D_{X} \mathscr{Y} Y-\mathrm{w}_{\mathscr{P}} \perp_{Y} X-\operatorname{th}(X, Y)+\right. \\
& +\left(\mathrm{D}_{\mathrm{X}} \mathscr{P}^{\mathcal{I}}\right) \mathrm{Y}+\mathrm{h}(\mathrm{X}, \mathscr{P} \mathrm{Y})-\mathrm{f} \mathrm{h}(\mathrm{X}, \mathrm{Y})
\end{aligned}
$$

Let us use (5.1) to substitute in (5.5); a comparisson between the normal components in (5.5) leads to (5.4), Q.E.D.

Now we may use the above lemma to end the proof of the involutivity of $\mathscr{D}_{\mathrm{N}}$. Indeed, by (5.4) and (2.4) our (5.3) turns into:

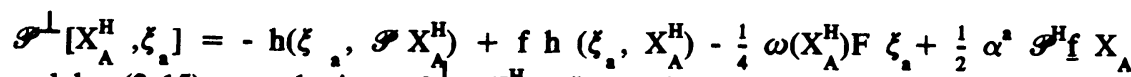
and by (2.15) one obtaines $\mathscr{S}^{\mathcal{A}}\left[\mathrm{X}_{\hat{\mathrm{H}}}^{\mathrm{H}}, \xi_{\mathrm{A}}\right]=\hat{0}$.

The last step is to establish minimality of $\mathscr{D}_{\mathbf{N}}^{\perp}$. Let $q=\operatorname{dim}_{\mathbb{R}} \mathscr{D}_{\mathbf{x}}^{\perp}, \mathrm{x} \in \mathbf{M}$. 
If $\left\{E_{i}: 1 \leq i \leq q\right\}$ is an orthonormal frame of $\mathscr{D}^{\perp}$ then (2.8) yields:

$$
l_{N} \sum_{i=1}^{q} \underline{D}_{E^{H}} \mathbf{E}_{i}^{H}=\left\{l \sum_{i=i}^{q} \underset{i}{\nabla_{i}} E^{-E_{i}^{H}}\right\} \text {. }
$$

But $\mathscr{D}^{\perp}$ is minimal, so the right hand member of (5.7) is zero. Finally, one may follow the ideas in [17], (p.170) to show that since $\mathscr{D}_{\mathrm{N}}$ is integrable and $\mathscr{D}_{\mathrm{N}}$ minimal the $(2 p+s)$-form $\lambda$ is coclosed. As $N$ is compact, $\lambda$ is harmonic. Thus $c(N)=[\lambda] \neq 0$, and our theor. $B$ is completely proved.

\section{REFERENCES}

1 A.BEJANCU, C.R. submanifolds of a Kaehler manifold, I-II, Proc. A.M.S. 69 (1978), 134-142. Trans. A.M.S. 250 (1979),335-345.

2 D.E.BLAIR \& B.Y.CHEN, On C.R. submanifolds of Hermitian manifolds, Israel L. Math. 34 (1979), 353-363

3 A.ANDREOTTI \& C.D.HILL, Complex characteristic coordinates and tangential Cauchy-Riemann equations, Ann. Scuola Norm. Sup. Pisa 26 (1972), 299-324.

4 D.E.BLAIR, Geometry of manifolds with structural group U(n) $\times O(s), L$ Diff. Geometry (2) 4 (1970), 155-167.

5 I.MIHAI, C.R. Submanifolds of a framed f-manifold, Stud. Cerc. Mat. 35 (1982), 127-136.

6 L.ORNEA, Generic C.R. submanifolds of $\mathscr{R}$ manifolds, Stud. Cerc. Mat. $\underline{36}$ (1984), 135-443.

7 K.YANO \& M.KON, C.R. submanifolds of Kaehlerian and Sasakian manifolds, Progress in Math. Vol, 30. Birkhäuser, Boston-Bascel-Stuttgart, 1983.

8 D.E.BLAIR, On a generalization of the Hopf fibration, An. St. Univ. "Al. I. Cuza". 17 (1971), 171-177.

9 W.M.BOOTHBY \& H.C.WANG, On contact manifolds, Ann. Math. (3) 68 (1958), 721-734.

10 A.MORIMOTO, On normal almost contact stuctures, L. Math. Soc. Japan, 15 (1963), 420-436.

11 S.TANNO, On fiberings of some noncompact contact manifolds, Tohoku Math. L. 15 (1963), 289-297.

12 I.VAISMAN, Locally conformal Kaehler manifolds with parallel Lee form, Rendiconti di Matem. 12 (1979), 263-284.

13 H.B. LAWSON, Rigidity theorems in rank-1 symmetric spaces, $L$ Diff. Geometry 4 (1970), 349-357.

14 Y.MAEDA, On real hypersurfces of a complex projective space, $L$ Math. Soc Japan 28 (1976), 529-540.

15 M.OKUMURA, On some real hypersurfaces of a complex projective space, Trans. A.M.S. 212 (1975), 355-364.

16 K.YANO \& M.KON, Differential geometry of C.R. submanifolds, Geom. Dedicata. 10 (1981), 369-391.

17 B.Y.CHEN, Cohomology of C.R. submanifolds, Ann. Fac. Sci. Toulose, 3 (1981), 167-172. 
18 S.GOLDBERG \& K.YANO, On normal globally framed f-manifolds, Tôhoku Math. J.2 22 (1970), 362-370.

19 K.YANO On a structure defined by a tensor field $f$ of type $(1,1)$ satisfying $\mathrm{f}^{3}+f=0$, Tensor. N.S.14 (1963), 99-109.

20 H.NAKAGAWA, f-structures induced on submanifolds in spaces, almost Hermitian or almost Kaehlerian, Ködai Math. Sem. Rep. 18 (1966), 161-183.

21 D.E.BLAIR \& G.D.LUDDEN \& K.YANO, Differential geometric stuctures on principal toroidal bundles, Trans.A.M.S. 181 (1973), 175-184.

22 R.S.PALAIS, A global formulation of the Lie theory of transformation groups. Memoirs A.M.S., vol.22, 1957.

23 B.O'NEILL, The fundamental equations of a submersion, Michigan Math. L. 13 (1966), 459-469.

24 B.Y.CHEN, Geometry of submanifolds. M.Dekker, Inc., New York, 1973.

25 S.GOLDBERG, Invariant submanifolds of codimension 2 of framed manifolds, An. St. Univ "Al. I. Cuza"'17 (1971), 157-165.

26 P.LIBERMANN, Sur les structures presque complexes et autres structures infinitesimales reguliers, Bull. Soc. Math. France. 83 (1985), 195-224.

27 B.Y.CHEN \& P.PICCINNI, The canonical foliations of a locally conformal Kaehler manifold, Ann. Matem. pura appl. (4) CXLI (1985), 289-305.

28 A.BEJANCU, Geometry of C.R. submanifolds D. Reidel Publ. Co., Dordrecht- Boston-Lancaster-Tokyo, 1986. 


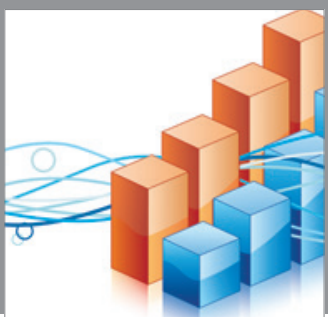

Advances in

Operations Research

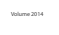

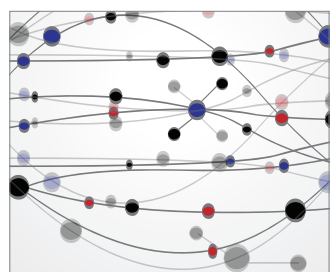

\section{The Scientific} World Journal
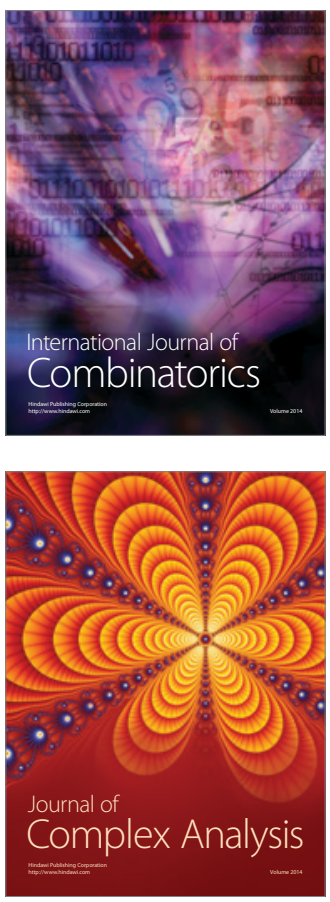

International Journal of

Mathematics and

Mathematical

Sciences
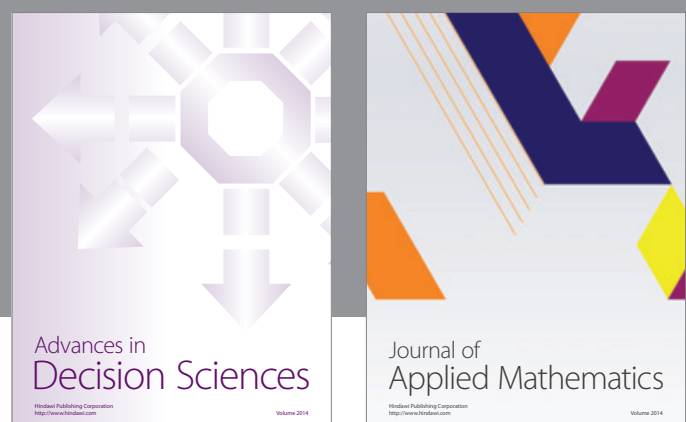

Journal of

Applied Mathematics
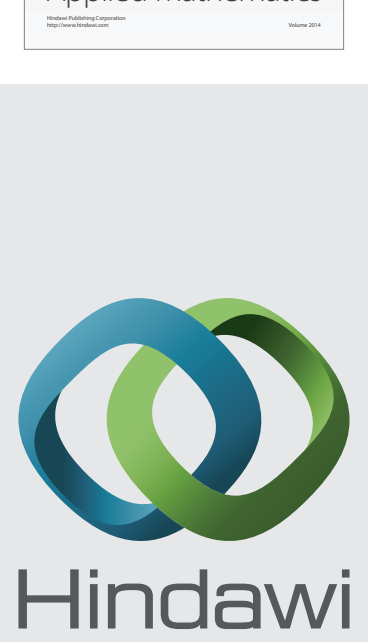

Submit your manuscripts at http://www.hindawi.com
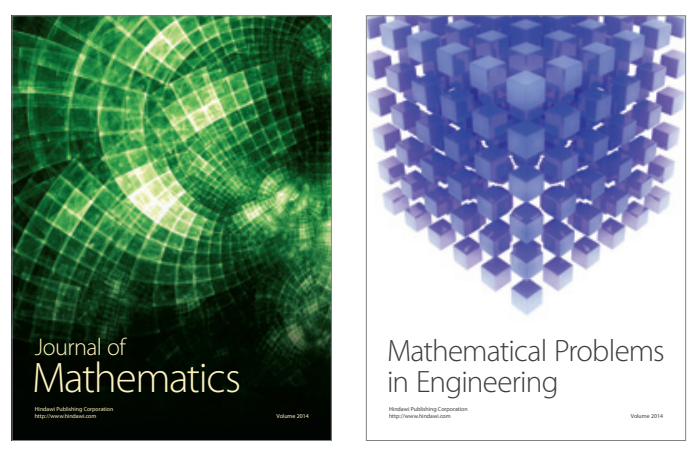

Mathematical Problems in Engineering
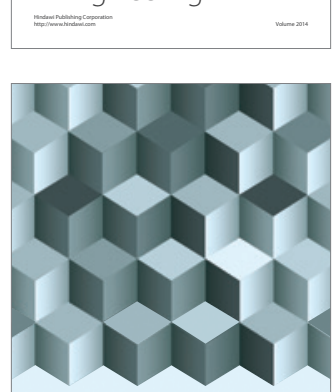

Journal of

Function Spaces
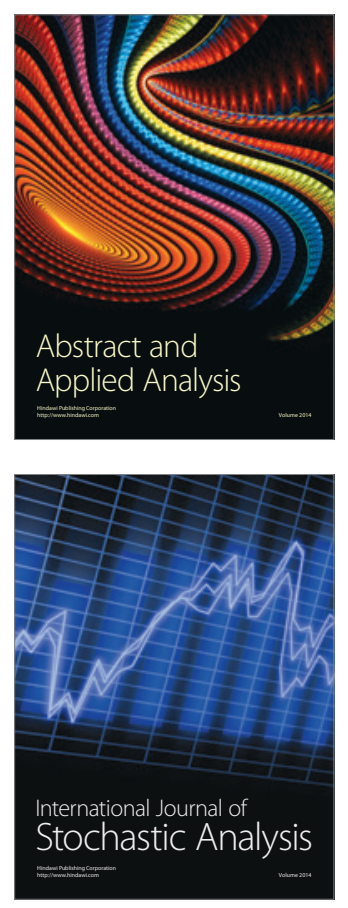

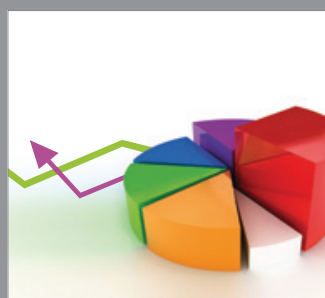

ournal of

Probability and Statistics

Promensencen
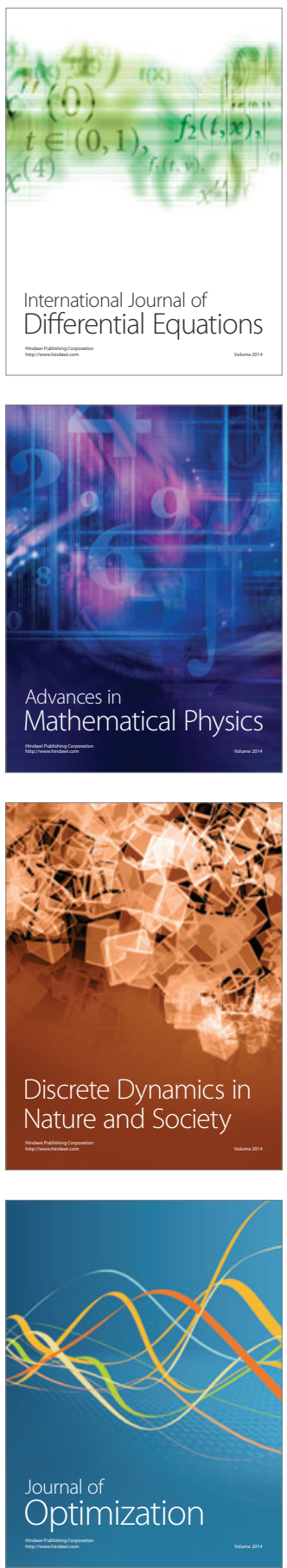